\title{
Butterfly Diradical Intermediates in Photochemical Reactions of $\mathrm{Fe}_{2}(\mathrm{CO})_{6}\left(\mu-\mathrm{S}_{2}\right)$

\author{
Ioan Silaghi-Dumitrescu, ${ }^{*}$ Thomas E. Bitterwolf, ${ }^{\dagger}$ and R. Bruce King ${ }^{\ddagger}$ \\ *Faculty of Chemistry and Chemical Engineering, Babes-Bolyai University, ${ }^{\dagger}$ Cluj-Napoca, \\ Romania; Department of Chemistry, University of Idaho, Moscow, Idaho; Department of Chemistry \\ and ${ }^{\ddagger}$ Center for Computational Chemistry, University of Georgia, Athens, Georgia 30606.
}

\section{Supporting Information}

Table S01. BB86/6-311G* and /Lanl2dz/6-31G* calculated energies (a.u) of $\mathrm{Fe}_{2} \mathrm{~S}_{2}(\mathrm{CO})_{6}$ clusters.

Table S02. BP86/6-311G* bond lengths $(\AA)$ in $\mathrm{Fe}_{2} \mathrm{~S}_{2}(\mathrm{CO})_{6}$ clusters

Cartesian Coordinates

Figures of all of the structures. 
Table S01. BB86/6-311G* and /Lanl2dz/6-31G* calculated energies (a.u) of $\mathrm{Fe}_{2} \mathrm{~S}_{2}(\mathrm{CO})_{6}$ clusters. Relative energies (in bold) are given in $\mathrm{kcal} / \mathrm{mol}$

\begin{tabular}{|c|c|c|c|c|}
\hline BP86 & $6-311 G(d)$ & $\begin{array}{l}\text { Imaginary } \\
\text { frequencies }\end{array}$ & Lanl2dz/6-31G(d) & $\begin{array}{l}\text { Imaginary } \\
\text { frequencies }\end{array}$ \\
\hline \multicolumn{5}{|l|}{ Singlet states } \\
\hline \multicolumn{5}{|l|}{ tetrahedrane } \\
\hline \multirow{2}{*}{$\begin{array}{rr}\mathbf{A} & \mathrm{C}_{2 \mathrm{v}} \\
& \mathrm{C}_{2}\end{array}$} & -4004.7128711 & i19.31 & -1723.7612171 & No \\
\hline & -4004.7129369 & No & -1723.7612159 & No \\
\hline Butterfly & \multirow[b]{2}{*}{-4004.6912108} & \multirow{3}{*}{ No } & \multirow[b]{2}{*}{-1723.7327575} & \multirow{3}{*}{ No } \\
\hline \multirow[t]{2}{*}{ B $\mathrm{FeFe} \quad \mathrm{C}_{2 \mathrm{v}}$} & & & & \\
\hline & A $\quad+\mathbf{1 3 . 6 3}$ & & A $\quad+\mathbf{1 7 . 8 6}$ & \\
\hline \multirow{3}{*}{$\begin{array}{llll}\mathbf{C} & \mathrm{SS} & \mathrm{C}_{2 \mathrm{v}} \\
& & \mathrm{C}_{2}\end{array}$} & \multirow{2}{*}{$\begin{array}{l}-4004.6503775 \\
-4004.6504149\end{array}$} & \multirow{3}{*}{$\begin{array}{l}\mathrm{i} 35.34 \\
\text { No }\end{array}$} & \multirow[t]{2}{*}{-1723.7002878} & \multirow[t]{3}{*}{ No } \\
\hline & & & & \\
\hline & $A \quad+39.23$ & & A $\quad+38.23$ & \\
\hline \multirow[t]{2}{*}{ Planar $\mathrm{Ci}$} & -4004.6654705 & \multirow[t]{2}{*}{ i 68.77TS } & -1723.7042619 & \multirow[t]{2}{*}{ i $92.87 \mathrm{TS}$} \\
\hline & B $\quad+16.15$ & & B $\quad+17.88$ & \\
\hline \multicolumn{5}{|l|}{ Triplet states } \\
\hline \multicolumn{5}{|l|}{ Butterfly } \\
\hline \multirow{2}{*}{$\begin{array}{l}\mathrm{Fe}-\mathrm{Fe} \\
\mathrm{C}_{2 \mathrm{v}}\end{array}$} & -4004.6757982 & \multirow[t]{2}{*}{ No } & -1723.721365 & \multirow[t]{2}{*}{ No } \\
\hline & $\mathrm{A} \quad+9.67$ & & $\mathrm{~A} \quad+7.15$ & \\
\hline S-S & -4004.6587572 & i7.6 & -1723.7111525 & No \\
\hline $\mathrm{C}_{2 \mathrm{v}}$ & C $\quad-5.23$ & & $\begin{array}{ll}C & -6.82 \\
\end{array}$ & \\
\hline & & & & \\
\hline
\end{tabular}

Table S02. BP86/6-311G* bond lengths $(\AA)$ in $\mathrm{Fe}_{2} \mathrm{~S}_{2}(\mathrm{CO})_{6}$ clusters.

\begin{tabular}{|c|c|c|c|c|}
\hline $\begin{array}{c}\text { Interatomic } \\
\text { distances }\end{array}$ & $\begin{array}{c}\text { Tetrahedrane } \\
C_{2 v}\end{array}$ & $\begin{array}{c}\text { Fe-Fe- } \\
\text { Butterfly } \\
C_{2 v}\end{array}$ & $\begin{array}{c}\text { S-S- } \\
\text { Butterfly } \\
C_{2 v}\end{array}$ & Planar $C_{i}$ \\
\hline $\mathrm{Fe}(1)-\mathrm{Fe}(2)$ & 2.503 & 2.746 & 3.645 & 3.375 \\
$\mathrm{Fe}(1)-\mathrm{S}(3)$ & 2.270 & 2.206 & 2.231 & 2.149 \\
$\mathrm{Fe}(1)-\mathrm{S}(4)$ & 2.270 & 2.206 & 2.231 & 2.334 \\
$\mathrm{Fe}(1)-\mathrm{C}(11)$ & 1.775 & 1.796 & 1.779 & 1.772 \\
$\mathrm{Fe}(1)-\mathrm{C}(13)$ & 1.761 & 1.741 & 1.775 & 1.773 \\
$\mathrm{Fe}(1)-\mathrm{C}(15)$ & 1.761 & 1.796 & 1.775 & 1.798 \\
$\mathrm{C}(11)-\mathrm{O}(12)$ & 1.157 & 1.154 & 1.158 & 1.155 \\
$\mathrm{C}(13)-\mathrm{O}(14)$ & 1.158 & 1.156 & 1.158 & 1.155 \\
$\mathrm{C}(15)-\mathrm{O}(16)$ & 1.158 & 1.154 & 1.158 & 1.153 \\
$\mathrm{~S}(3)-\mathrm{S}(4)$ & 2.083 & 3.139 & 2.160 & 2.957 \\
\hline
\end{tabular}


Cartesian coordinates

\#P BP86/6-311G* SCF=(DIRECT MAXCYCLE=256) OPT=CARTESIAN

$\mathrm{Fe}_{2}(\mathrm{CO})_{6} \mathrm{~S}_{2} C_{2 v}, \mathrm{Fe}-\mathrm{Fe}$ butterfly State=1-A1\HF=-4004.6912108
01
$\mathrm{Fe} \quad 1.3731454562 \quad 0 . \quad-0.0097210356$
S $\quad 0 . \quad 1.5696739856 \quad-0.7272794968$
Fe $-1.3731454562 \quad 0 .-0.0097210356$
S $\quad 0 . \quad-1.5696739856 \quad-0.7272794968$
$\begin{array}{llll}\text { C } & -2.5272144946 & 0 . & -1.3129729378\end{array}$
$\begin{array}{llll}\text { C } & -2.1096217681 & 1.3126378413 & 0.9698452003\end{array}$
$\begin{array}{llll}\text { C } & -2.1096217681 & -1.3126378413 & 0.9698452003\end{array}$
$\begin{array}{llll}\text { C } & 2.1096217681 & -1.3126378413 & 0.9698452003\end{array}$
$\begin{array}{llll}\text { C } & 2.1096217681 & 1.3126378413 & 0.9698452003\end{array}$
$\begin{array}{llll}\text { C } & 2.5272144946 & 0 . & -1.3129729378\end{array}$
O $\quad-3.2658284076$ 0. -2.2016694692
$\begin{array}{llll}\text { O } & -2.5857563028 & -2.1470480662 & 1.6088918658\end{array}$
$\begin{array}{llll}\text { O } & -2.5857563028 & 2.1470480662 & 1.6088918658\end{array}$
$\begin{array}{lllll}\text { O } & 3.2658284076 & 0 . & -2.2016694692\end{array}$
$\begin{array}{llll}\text { O } & 2.5857563028 & 2.1470480662 & 1.6088918658\end{array}$
$\begin{array}{llll}\text { O } & 2.5857563028 & -2.1470480662 & 1.6088918658\end{array}$

\#P BP86/6-311G(d) scf=direct Pop=full geom=checkpoint

$\mathrm{Fe}_{2}(\mathrm{CO})_{6} \mathrm{~S}_{2}$ Tetrahedrane $C_{2 v}$ optim $6-311 \mathrm{G}^{*}-4004.7128711$
01
S $\quad-1.0415734414 \quad 0.0000000000 \quad-1.6420266314$
$\begin{array}{llll}\mathrm{Fe} & 0.0000000000 & 1.2513137293 & -0.0602073777\end{array}$
$\begin{array}{llll}\text { C } & 1.2986943159 & 1.5028941902 & 1.1017250542\end{array}$
$\begin{array}{llll}\text { C } & -1.2986943159 & 1.5028941902 & 1.1017250542\end{array}$
$\begin{array}{llll}\text { C } & 0.0000000000 & 2.8602006484 & -0.8092484612\end{array}$
$\begin{array}{llll}\mathrm{Fe} & 0.0000000000 & -1.2513137293 & -0.0602073777\end{array}$
C $\quad 0.0 \quad-2.8602006484 \quad-0.8092484612$
C $\quad-1.2986943159 \quad-1.5028941902 \quad 1.1017250542$
$\begin{array}{llll}\text { S } & 1.0415734414 & 0.0 & -1.6420266314\end{array}$
C $1.2986943159-1.5028941902 \quad 1.1017250542$
$\begin{array}{llll}\text { O } & 0.0 & -3.9210376356 & -1.271123582\end{array}$
$\begin{array}{llll}\mathrm{O} & 0.0 & 3.9210376356 & -1.271123582\end{array}$
$\begin{array}{llll}\text { O } & 2.1629011171 & 1.6797031731 & 1.8525997934\end{array}$
$\begin{array}{llll}\text { O } & -2.1629011171 & 1.6797031731 & 1.8525997934\end{array}$
$\begin{array}{llll}\text { O } & -2.1629011171 & -1.6797031731 & 1.8525997934\end{array}$
$\begin{array}{llll}\text { O } & 2.1629011171 & -1.6797031731 & 1.8525997934\end{array}$

\#P BP86/6-311G* SCF=(DIRECT, MAXCYCLE=256) OPT=CARTESIAN 
$\mathrm{Fe}_{2}(\mathrm{CO})_{6} \mathrm{~S}_{2}$ planar $C_{i}$ State=1-AG $\backslash H F=-4004.6654705$
01
S $\quad 0.0257049343 \quad-1.4637752705 \quad-0.2072034622$
C $1.261687615 \quad 1.1238737159 \quad-2.3929362703$
$\begin{array}{llll}\text { O } & 0.0409497792 & -1.9880955246 & -3.772594567\end{array}$
$\begin{array}{llll}\text { O } & -2.216932384 & 1.6535688333 & -2.7847533904\end{array}$
$\begin{array}{lllll}\text { O } & 2.1435658851 & 1.7512857187 & -2.797551317\end{array}$
Fe $0.00178309150 .0970474662 \quad-1.6848653911$
C $0.0288972928-1.1680392912-2.962636762$
Fe $\quad-0.0017830915 \quad-0.0970474662 \quad 1.6848653911$
$\begin{array}{llll}\text { S } & -0.0257049343 & 1.4637752705 & 0.2072034622\end{array}$
$\begin{array}{llll}\text { C } & -0.0288972928 & 1.1680392912 & 2.962636762\end{array}$
$\begin{array}{llll}\text { C } & -1.3054450262 & 1.0647750805 & -2.3877351794\end{array}$
$\begin{array}{llll}\text { C } & -1.261687615 & -1.1238737159 & 2.3929362703\end{array}$
C $1.3054450262-1.0647750805 \quad 2.3877351794$
$\begin{array}{llll}\text { O } & -2.1435658851 & -1.7512857187 & 2.797551317\end{array}$
$\begin{array}{lllll}\text { O } & -0.0409497792 & 1.9880955246 & 3.772594567\end{array}$
$\begin{array}{lllll}\text { O } & 2.216932384 & -1.6535688333 & 2.7847533904\end{array}$

\#P BP86/6-311G(d) opt freq

$\mathrm{Fe}_{2}(\mathrm{CO})_{6} \mathrm{~S}_{2}$ SS Butterfly $C_{2 v}$ HF=-4004.6503776

$\begin{array}{llll}01 & & & \\ \mathrm{Fe} & -1.3862680611 & 0.1405578118 & -1.1850335582 \\ \mathrm{~S} & -0.3903069485 & 1.2260657689 & 0.4905778936 \\ \mathrm{Fe} & 1.4662771645 & 0.1405578118 & 1.0844698928 \\ \mathrm{~S} & 0.832647313 & 0.3281172203 & -1.046556733 \\ \mathrm{C} & 0.9520972877 & -1.4764658046 & 1.6200023853 \\ \mathrm{C} & 1.7216344382 & 0.929925514 & 2.6535644503 \\ \mathrm{C} & 3.1942153121 & -0.151310228 & 0.8026734375 \\ \mathrm{C} & -1.5000305655 & -0.151310228 & -2.9320989768 \\ \mathrm{C} & -2.9726114393 & 0.929925514 & -1.081207964 \\ \mathrm{C} & -1.7925394063 & -1.4764658046 & -0.5636483393 \\ \mathrm{O} & 0.6785243012 & -2.5292884352 & 2.0173737624 \\ \mathrm{O} & 4.303506923 & -0.3911088315 & 0.5703765673 \\ \mathrm{O} & 1.8194626323 & 1.4327892779 & 3.6925787734 \\ \mathrm{O} & -2.1182600028 & -2.5292884352 & -0.2077659384 \\ \mathrm{O} & -4.0070313397 & 1.4327892779 & -0.9430175559 \\ \mathrm{O} & -1.522987049 & -0.3911088315 & -4.065219762\end{array}$

\section{\#P RBP86/6-311G(D) OPT FREQ}

$\mathrm{Fe}_{2} \mathrm{~S}_{2}(\mathrm{CO})_{5}$ from $\mathrm{SS}$ butterfly $\mathrm{Fe}_{2} \mathrm{~S}_{2}(\mathrm{CO})_{6} C_{2 v} \quad \mathrm{HF}=-3891.2906687$

$\begin{array}{ll}0 & 1\end{array}$ 
Fe $\quad-1.1572280213 \quad 0.0278359358 \quad-0.113822279$

S $\quad 0.3616114021 \quad 0.3202232513 \quad-1.606548272$

S $\quad 0.2028745359 \quad-1.5730336825 \quad 0.9821926948$

Fe $1.411458285 \quad-0.1033359843 \quad 0.1705736291$

C $\quad-2.3177705913 \quad-1.2961333389 \quad 0.1027278836$

$\begin{array}{llll}\text { O } & -3.1074274779 & -2.1347590051 & 0.1815728158\end{array}$

C $\quad-1.4666969987 \quad 0.8316083301 \quad 1.4483191534$

$\begin{array}{llll}\text { O } & -1.61929882 & 1.3891738544 & 2.4480145751\end{array}$

C $\quad-2.234405985 \quad 1.004371696 \quad-1.1243045715$

$\begin{array}{llll}\text { O } & -2.9599010966 & 1.6179568407 & -1.7808370473\end{array}$

C $2.8854889007 \quad-0.9445780118 \quad-0.1496051322$

$\begin{array}{llll}\text { O } & 3.8431172975 & -1.541692057 & -0.4181217398\end{array}$

C $\quad 2.1544498669 \quad 1.5253626026 \quad 0.1937460234$

$\begin{array}{llll}\text { O } & 2.6224909692 & 2.5798429286 & 0.2804781451\end{array}$ 